\title{
Performance of the LHCb Silicon Tracker
}

\author{
Greig Cowan
}

On behalf of the LHCb Silicon Tracker group

HSTD-8, 7th December 201

$1 \mathrm{LHCb}$ tracker

2 Operational experience

3 Alignmept

4 Detector efficiency and resblution

5 Radiation damage

6 Summary

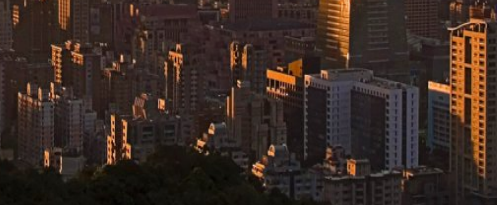

(PFI

ECOLE POLYTECHNIOU FÉDÉRALE DE LAUSANNE

rhap $\rightarrow-\cdots$ 


\section{LHCb detector}

- Forward arm spectrometer. $b / \bar{b}$ production correlated in forward/backward direction.

- Access to $B_{u}, B_{d}, B_{s}, B_{c}, \Lambda_{b}$.

- Excellent tracking and momentum res for good mass res. $\delta p / p \sim 0.4 \%$.
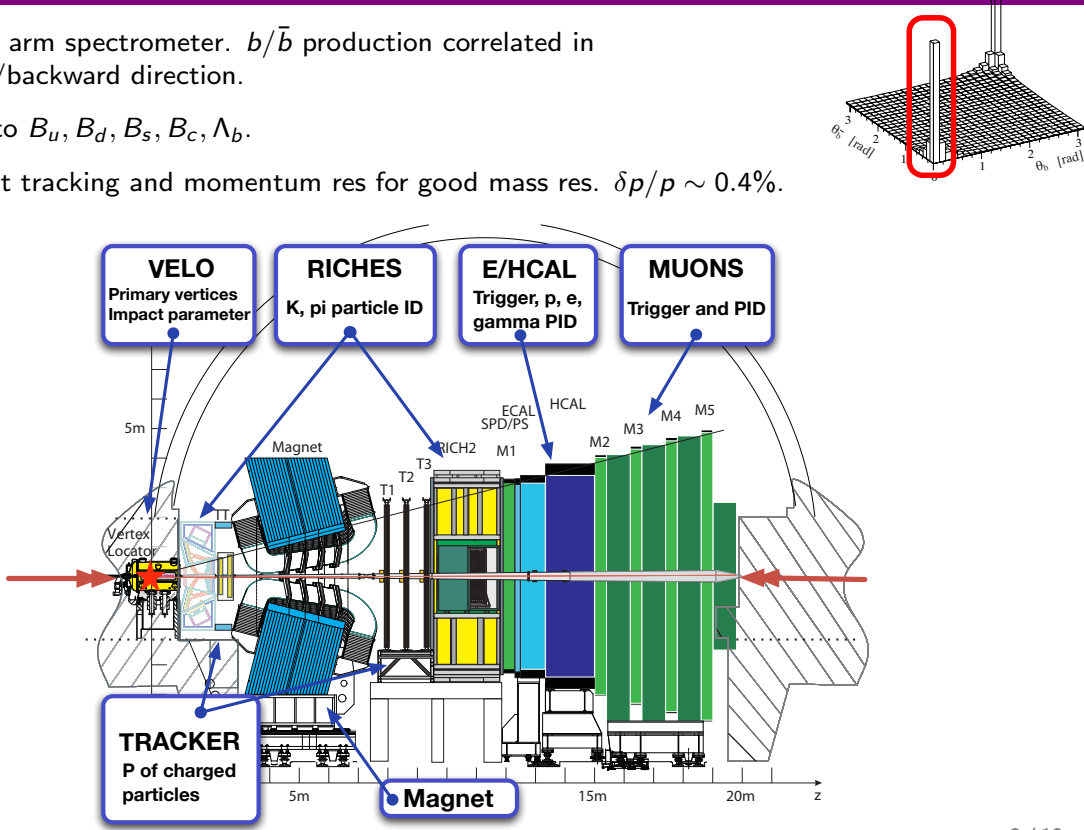


\section{LHCb detector}

- Forward arm spectrometer. $b / \bar{b}$ production correlated in forward/backward direction.

- Access to $B_{u}, B_{d}, B_{s}, B_{c}, \Lambda_{b}$.

- Excellent tracking and momentum res for good mass res. $\delta p / p \sim 0.4 \%$.
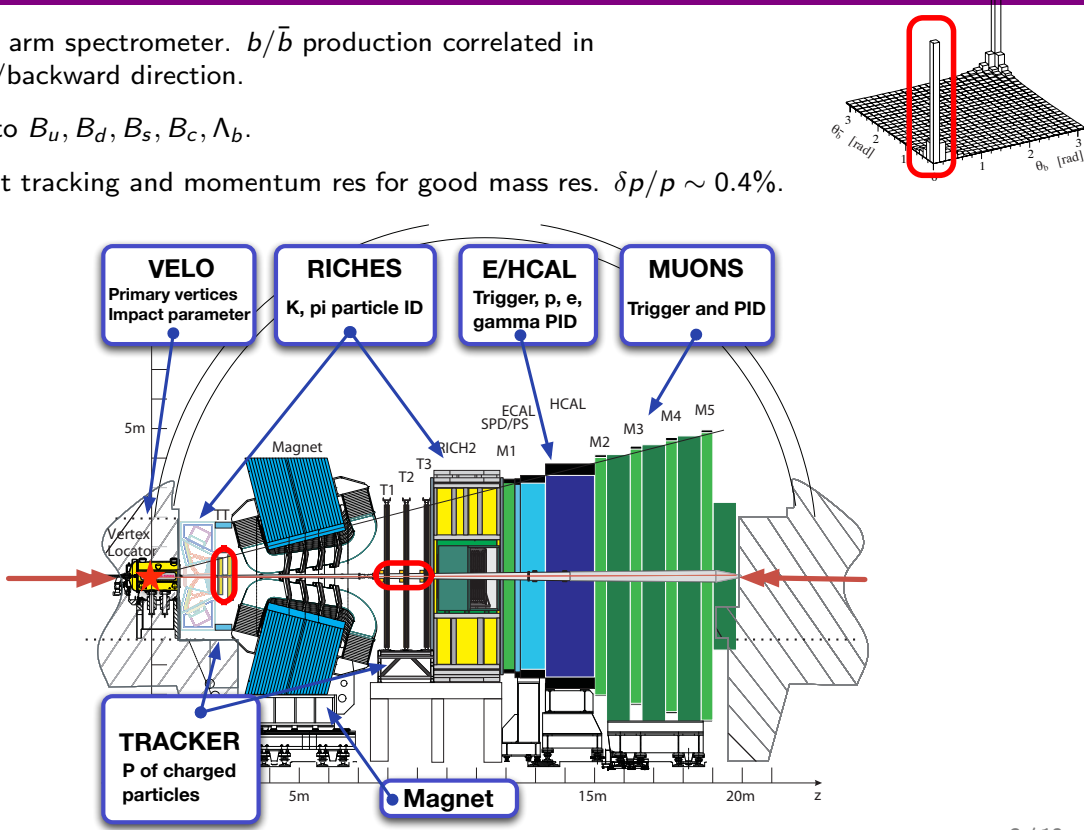


\section{Tracking at $\mathrm{LHCb}$}

1 Charged particles get kick from dipole magnet - momentum information.

2 Reconstruct primary and secondary vertices.

3 Extrapolation to RICH, CALO and Muon systems for particle ID.

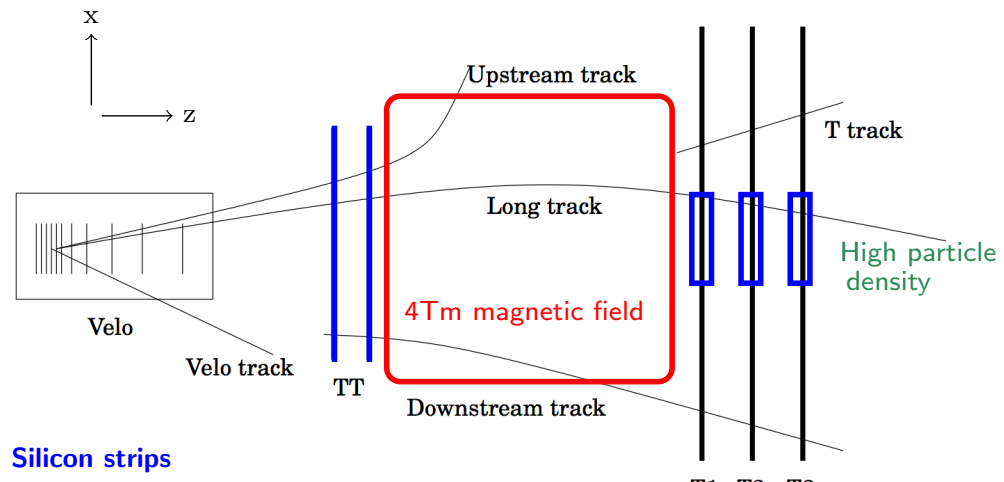

T1 T2 T3

See following talk from P. Collins for details about the VELO 


\section{Tracker Turencisis (TT)}
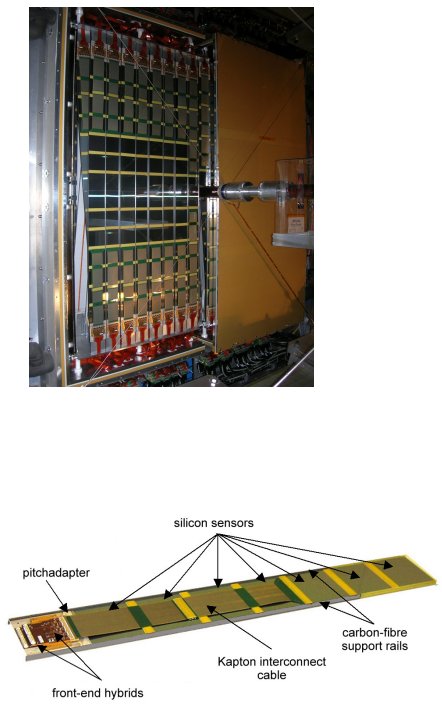

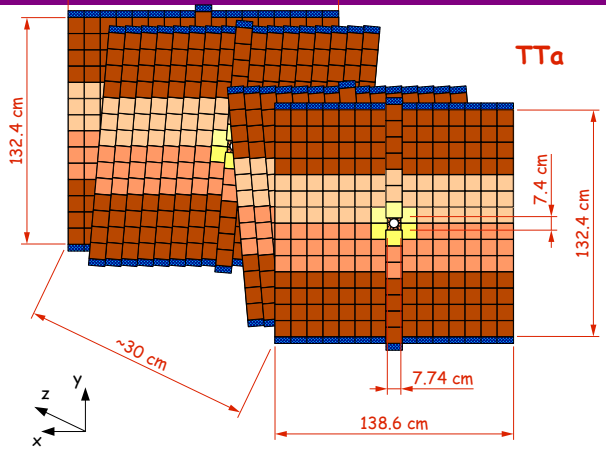

- Four planes of p-on-n "HPK" silicon $\left(0^{\circ},+5^{\circ},-5^{\circ}, 0^{\circ}\right)$.

- $500 \mu \mathrm{m}$ thick, $183 \mu \mathrm{m}$ pitch.

- Long strips, up to $37 \mathrm{~cm}$.

- Total area $8.2 \mathrm{~m}^{2}, 143 \mathrm{k}$ strips.

- Complete station is $7 \%$ of a radiation length.

- Temperature: $8^{\circ} \mathrm{C}$ for sensors, $0^{\circ} \mathrm{C}$ coolant.

- Digitisation in off-detector service box. 


\section{Inner tracker (IT)}
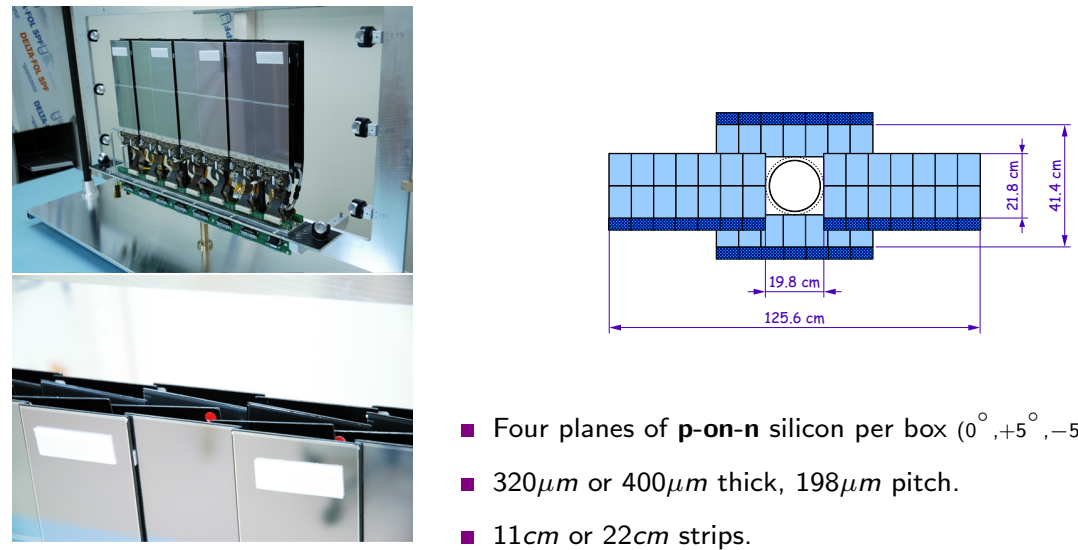

- Four planes of p-on-n silicon per box $\left(0^{\circ},+5^{\circ},-5^{\circ}, 0^{\circ}\right)$.

- $320 \mu \mathrm{m}$ or $400 \mu \mathrm{m}$ thick, $198 \mu \mathrm{m}$ pitch.

- $11 \mathrm{~cm}$ or $22 \mathrm{~cm}$ strips.

- Total area $4 \mathrm{~m}^{2}, 130 \mathrm{k}$ strips.

two silicon sensors

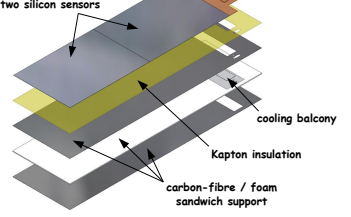

- 3 stations each have $4 \%$ of a radiation length.

- Temperature: $10^{\circ} \mathrm{C}$ for sensors, $0^{\circ} \mathrm{C}$ coolant.

- Digitisation in off-detector service box (outside acceptance). 


\section{Operational status}
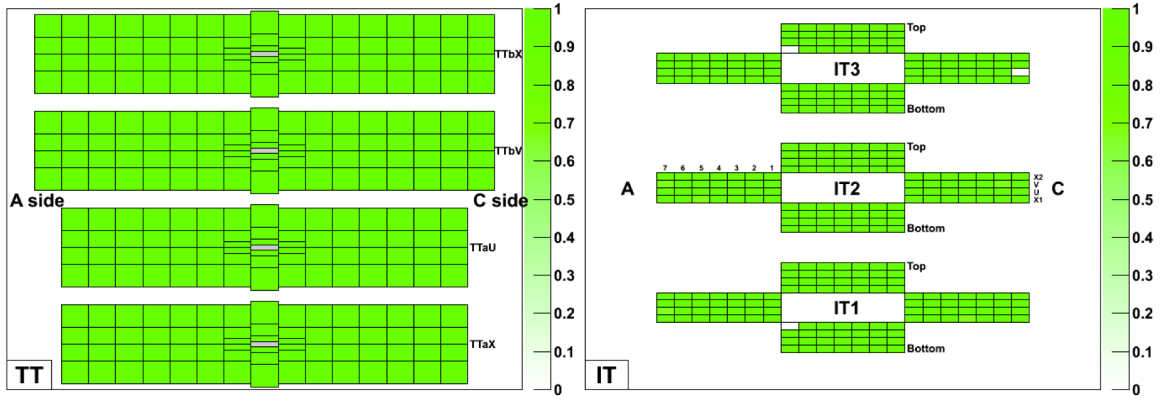

\section{TT}

\section{IT (more difficult to access)}

- 143360 readout channels.

- $99.77 \%$ working channels.

- 3 ports disabled.
- 129024 readout channels.

- $98.22 \%$ working channels.

- 10 ports disabled.

- 2 modules not configurable.

- 1 module with HV problem (will be fixed during winter). 


\section{Timing calibration (1)}

\section{Optimise charge collection}

- Different cable lengths for different detector parts.

- Time of flight different per station.

- Trigger and control signals must be synchronized for the entire $\mathrm{LHCb}$ detector.

\section{Time delay scan for each service box}

- Read out samples spaced by $25 n s$.

- Fit Landau $\otimes$ Gaussian to charge distribution for each sample.

- Shift sampling point by $-6,+6,+12 n s$.

\section{Synchronised scenario}

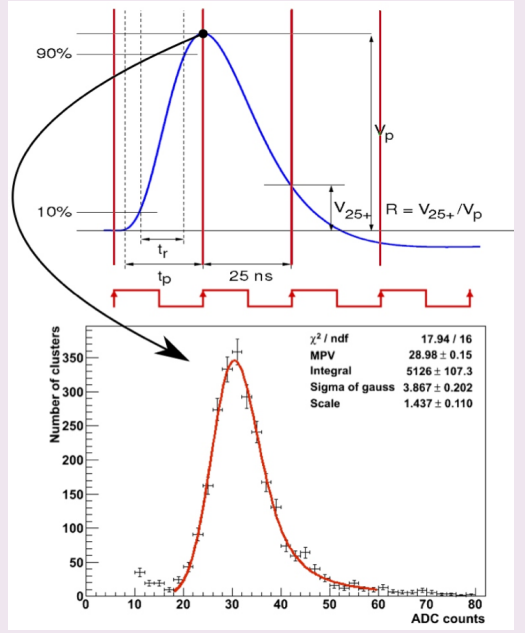




\section{Timing calibration (2)}

\section{Optimise charge collection}

- Different cable lengths for different detector parts.

- Time of flight different per station.

- Trigger and control signals must be synchronized for the entire $\mathrm{LHCb}$ detector.

\section{Time delay scan for each service box}

- Read out samples spaced by $25 n s$.

- Fit Landau $\otimes$ Gaussian to charge distribution for each sample.

- Shift sampling point by $-6,+6,+12 n s$.
- Plot MPV vs sample time.

- Fit pulse shape to extract optimal timing.

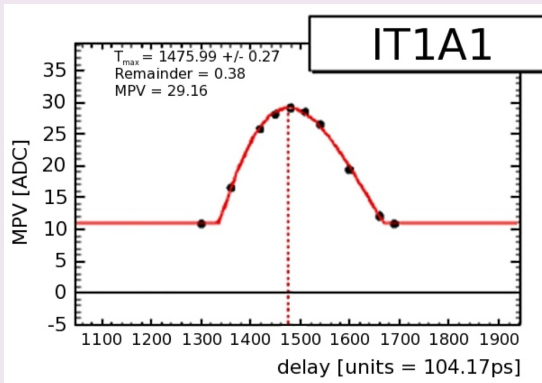

Collision data used to internally align TT and IT with resolution $<1$ ns 


\section{Spatial alignment}

- Tracking stations aligned using tracks which have been fitted with a Kalman filter W. Hulsbergen (NIM A600, 471).

- $\chi^{2}$ minimization of all track residuals using mass constraints.

- i.e., use $J / \psi \rightarrow \mu \mu$ or $D^{0} \rightarrow K \pi$ daughter tracks.

- Fix their origin and the invariant mass of the $J / \psi$ or $D^{0}$ to known value.

- Compute new tracks and residuals which go into $\chi^{2}$ minimization.

- Metrology survey constraints are used to fix certain degrees of freedom.
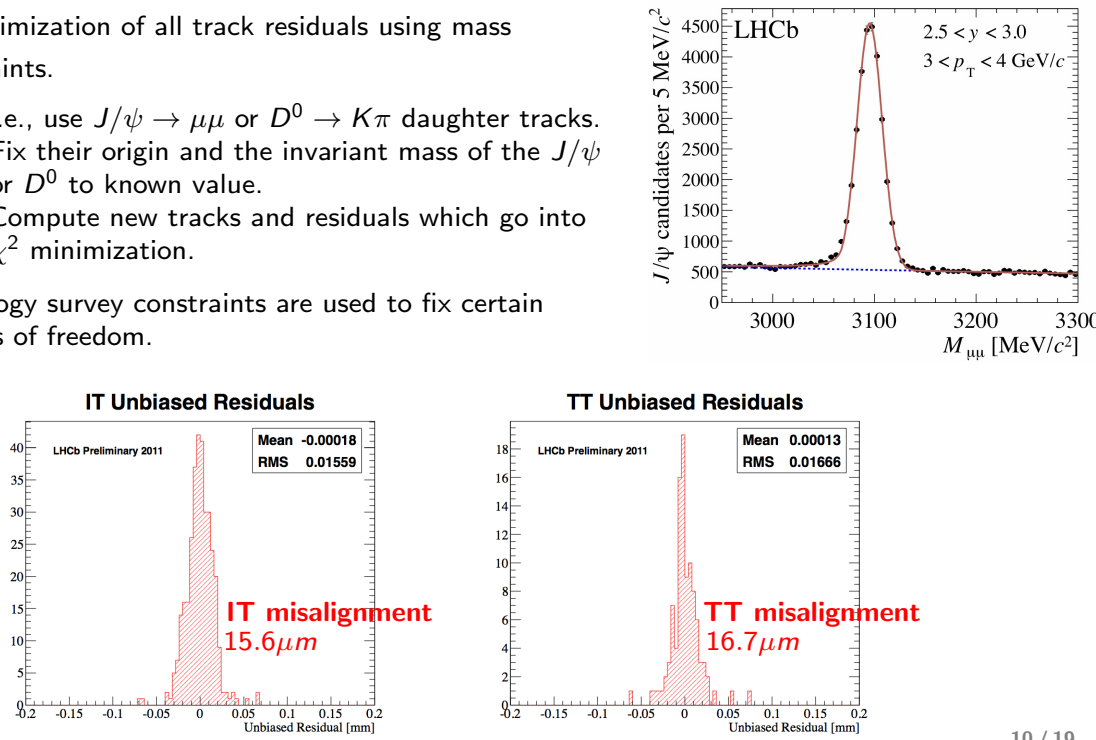


\section{Signal-noise performance}

- Measure $\mathrm{S} / \mathrm{N}$ for clusters assigned to tracks with $p>5 \mathrm{GeV}$.
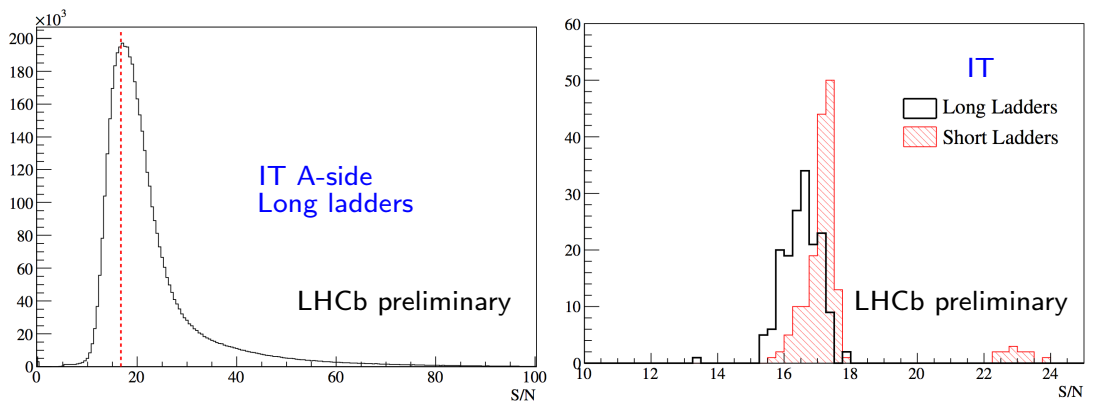

- S/N results:

- TT: $12-15$

- IT (long): 16.5

- IT (short): 17.5

- Within $10-20 \%$ of expectations from test beam. 


\section{Hit efficiency}

- Use daughter tracks from $D^{0} \rightarrow K K$ decays.

- Gives us a very pure sample of tracks, $p_{\text {track }}>10 \mathrm{GeV}$.

- For each track, extrapolate where we expect them in the silicon. Use isolation criteria.

- Within some search window, $\epsilon=\frac{\# \text { hits found }}{\# \text { \#its expected }}$.

- Two sectors have high common mode noise $(\epsilon>98 \%)$.
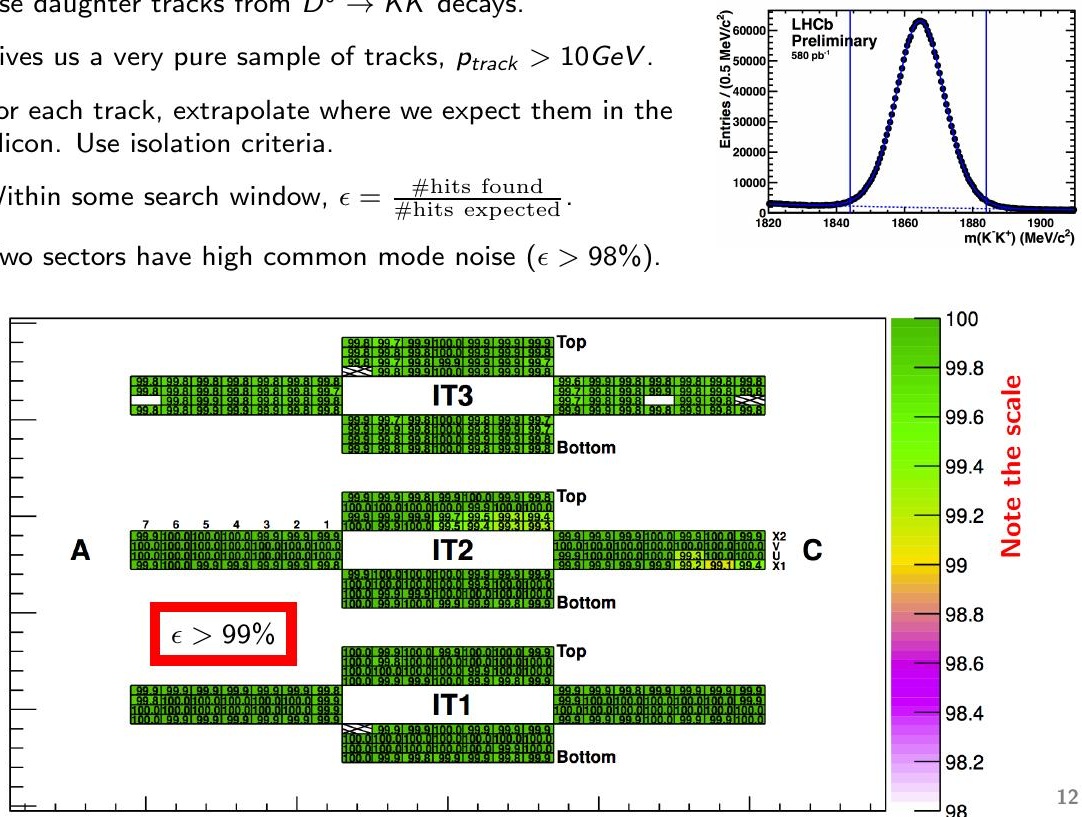


\section{Intrinsic charge sharing}

- Multi-strip clusters due to charge sharing.

- Interstrip track position: $\mathrm{x}$.

- Centre of gravity: $\eta=\mathrm{C}_{\mathrm{R}}(\mathrm{x}) /\left(\mathrm{C}_{\mathrm{L}}(\mathrm{x})+\mathrm{C}_{\mathrm{R}}(\mathrm{x})\right)$.
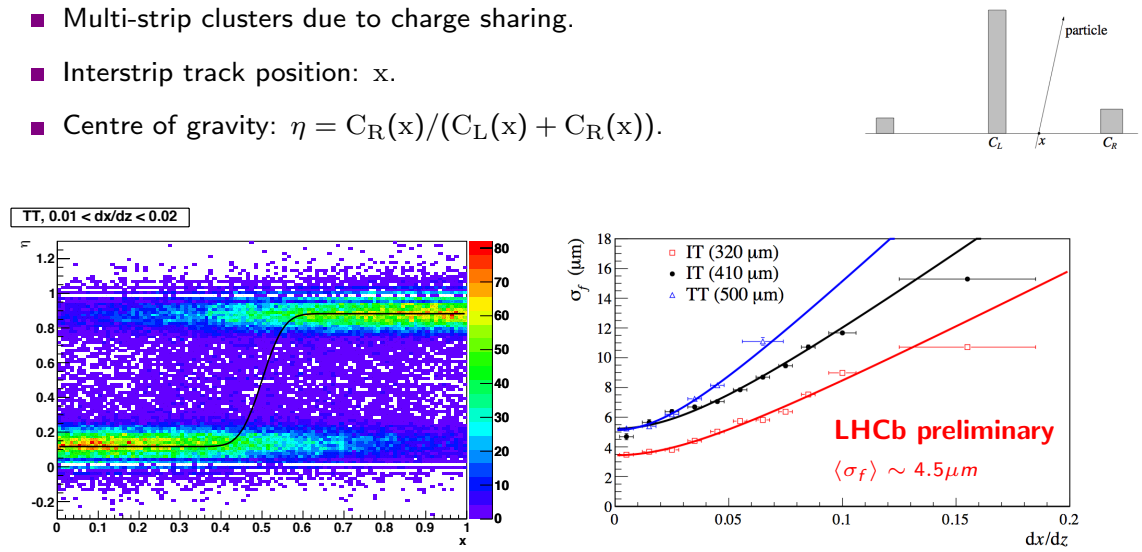

- Convolve error function with Gaussian in x (for mis-alignments) and $\eta$ (due to sharing).

- Perform 2D fit allows us to extract the charge sharing width $\left(\sigma_{f}\right)$ and track resolution.

- Black curve shows 1D cluster position shape: $\mathrm{f}\left(\mathrm{x} ; \sigma_{\mathrm{f}}\right)=\frac{1}{2}\left(1+\operatorname{erf}\left(\frac{\mathrm{x}-1 / 2}{\sqrt{2} \sigma_{\mathrm{f}}}\right)\right)$. 


\section{Radiation damage (1)}

LHCb Integrated Luminosity at $3.5 \mathrm{TeV}$ in 2011

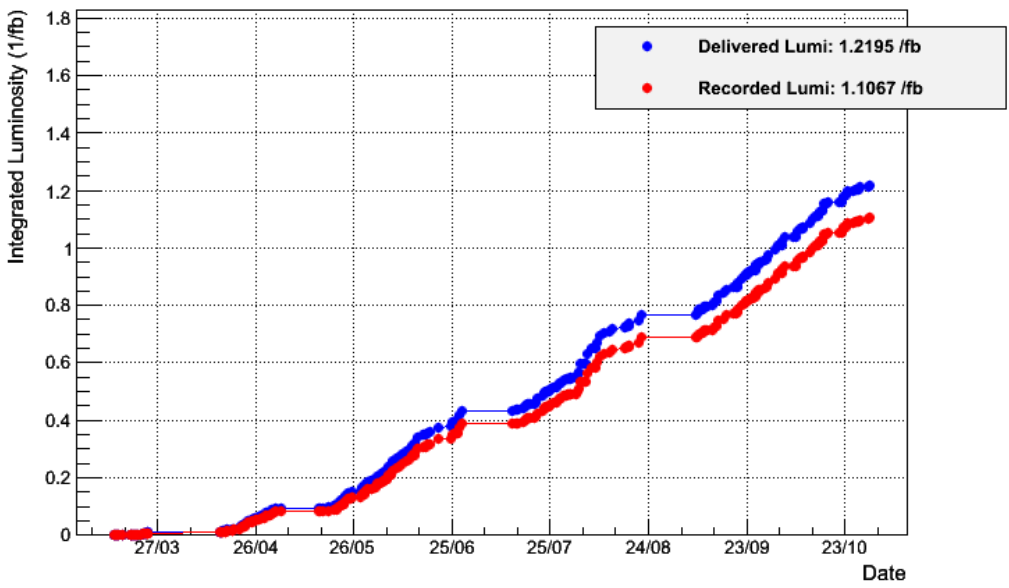

- LHCb designed to operate at lower luminosity than ATLAS/CMS! 


\section{Radiation damage (2)}

- Measure change in the leakage current and extract fluence.

- $\Delta I=\alpha \phi_{\text {eq }} V, \alpha=4 \times 10^{-17} \mathrm{~A} /$ particle $/ \mathrm{cm}^{-2}$

- Compare to what is expected from simulation:

\section{Expected fluence (FLUKA)}

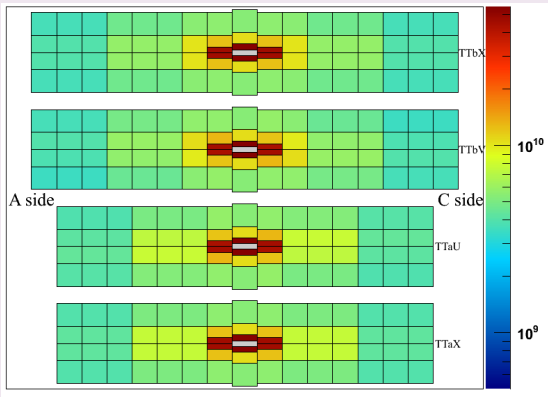

- Highest expected radiation dose (10 years):

- TT $=5 \times 10^{14} 1 \mathrm{MeV}$ neutron equiv.

- IT $=9 \times 10^{12} 1 \mathrm{MeV}$ neutron equiv.
Measured fluence $\left(2010, \int \mathcal{L}=45 p b^{-1}\right)$

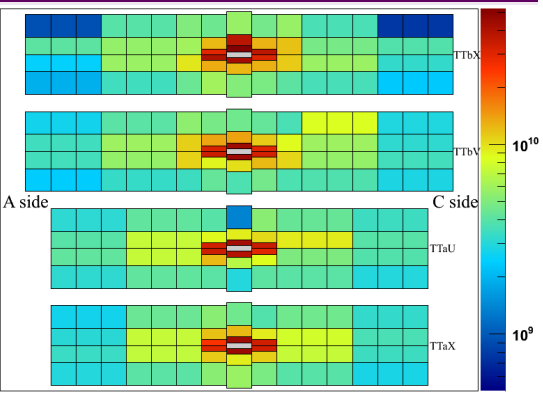




\section{Leakage current evolution (TT)}

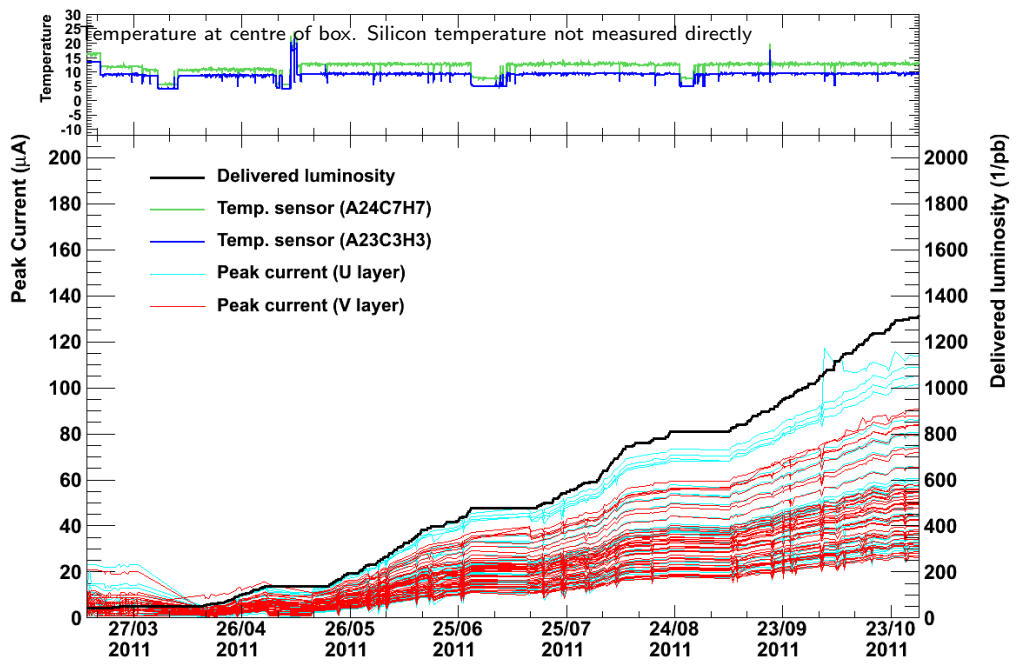

- Signs of annealing taking place during LHC technical stops.

- Current behaving as expected. 


\section{Leakage current evolution (IT)}
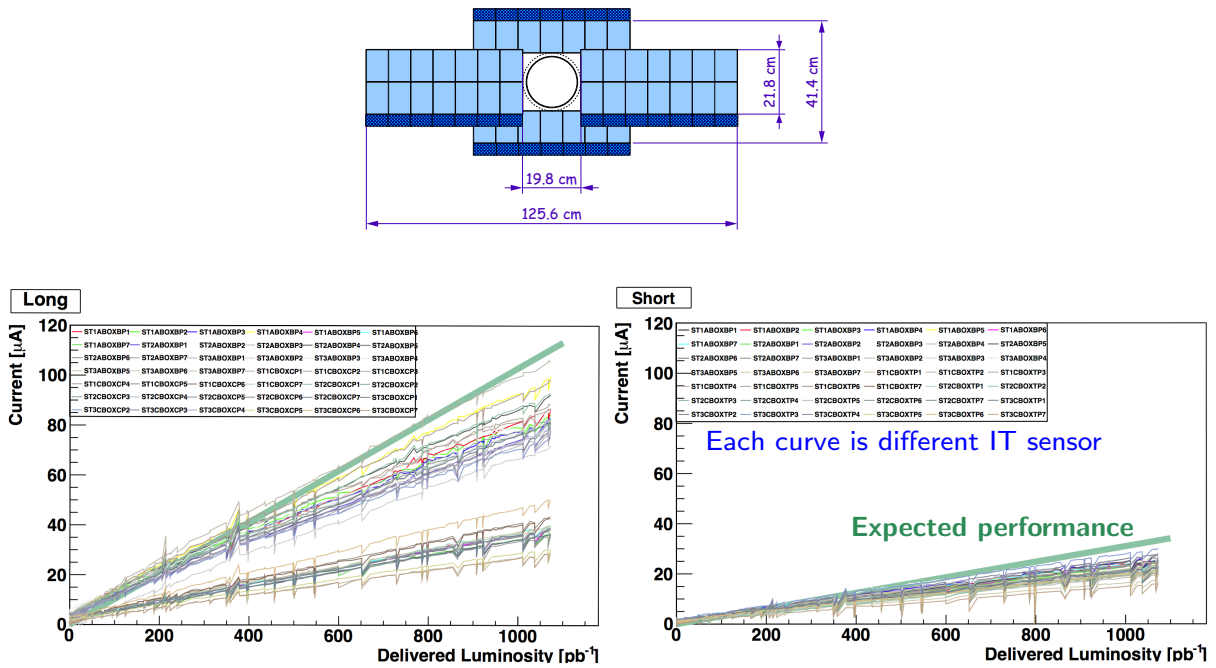

- Evolution of current with delivered luminosity behaving as expected. 


\section{Summary}

- LHCb silicon tracker is operating successfully at the LHC.

- > $99 \%$ and $>98 \%$ operational for TT and IT.

- Will use winter technical stop to perform some small improvements.

- Radiation damage being studied and is compatible with expectations.

- Performance is excellent.

- Time alignment $<1$ ns.

- $\mathrm{S} / \mathrm{N}$ is $\sim 14$ (TT), $\sim 17(\mathrm{IT})$.

- Spatial alignment to $\sim 16 \mu \mathrm{m}$.

- Hit efficiency is $>99 \%$ for IT.

$\Rightarrow$ Excellent physics performance of $\mathrm{LHCb}$ ! 


\section{Backup: Tracker readout chain}

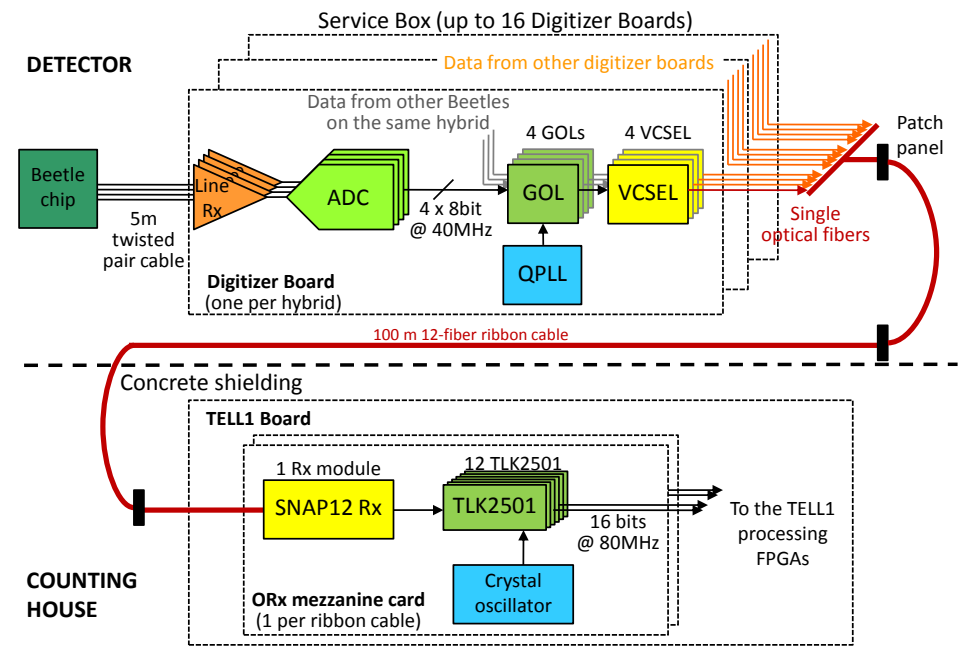

